\title{
Hemophilia A Pseudoaneurysm in a Patient with High Responding Inhibitors Complicating Total Knee Arthroplasty: Embolization: A Cost-Reducing Alternative to Medical Therapy
}

\author{
Ralph Kickuth, ${ }^{1}$ Suzanne Anderson, ${ }^{1}$ Kristiina Peter-Salonen, ${ }^{2}$ Bernhard Lämmle, ${ }^{2}$ Stefan Eggli, ${ }^{3}$ \\ Jürgen Triller ${ }^{1}$
}

${ }^{1}$ Institute of Diagnostic, Interventional and Pediatric Radiology, Inselspital, University of Berne, Berne, Switzerland

${ }^{2}$ Department of Hematology, Inselspital, University of Berne, Berne, Switzerland

${ }^{3}$ Department of Orthopedic Surgery, Inselspital, University of Berne, Berne, Switzerland

\begin{abstract}
Joint hemorrhages are very common in patients with severe hemophilia. Inhibitors in patients with hemophilia are allo-antibodies that neutralize the activity of the clotting factor. After total knee replacement, rare intra-articular bleeding complications might occur that do not respond to clotting factor replacement. We report a 40-year-old male with severe hemophilia A and high responding inhibitors presenting with recurrent knee joint hemorrhage after bilateral knee prosthetic surgery despite adequate clotting factor treatment. There were two episodes of marked postoperative hemarthrosis requiring extensive use of subsititution therapy. Eleven days postoperatively, there was further hemorrhage into the right knee. Digital subtraction angiography diagnosed a complicating pseudoaneurysm of the inferior lateral geniculate artery and embolization was successfully performed. Because clotting factor replacement therapy has proved to be excessively expensive and prolonged, especially in patients with inhibitors, we recommend the use of cost-effective early angiographic embolization.
\end{abstract}

Key words: Digital subtraction angiography-EmbolizationHemarthrosis-Hemophilia-Inhibitors-Interventional radiology-Pseudoaneurysm

Arterial bleeding as an exceptional complication following total knee arthroplasty (TKA) is an extremely rare entity in general, with an incidence of approximately $0.03 \%$ [1-3]. Pathologically, lacerations, pseudoaneurysms, and arteriovenous fistula might occur; most commonly, intrinsic (iatrogenic) as well as extrinsic risk factors are supposed to be causative for these bleeding phenomena [2]. In this context, hemophilia with high inhibitor titers is an essential predisposing factor for the acute onset of profuse life- or limb-threatening hemorrhage, especially in the postoperative phase [4-9]. High-dose clotting factor replacement therapy is the first-line treatment in hemophilic patients with inhibitors and postoperative episodes of bleeding $[10,11]$. High costs, occasional

Correspondence to: Ralph Kickuth; email: ralph.kickuth@insel.ch ineffectiveness in patients with inhibitors, and thrombembolic complications are potential disadvantages of this therapy [12]. Surgical treatment is an option in hemophiliacs with active bleeding after arthroplasty [7, 8]. Nevertheless, there are situations in which a surgical approach might be catastrophic-for example, failure of surgical attempts to stop bleeding, the inability to maintain satisfactory hematocrit levels, developing coagulation problems despite adequate substitution, or provocation of infections [13].

The role of transcatheter arterial embolization (TAE) in the therapy of hemophilic pseudoaneurysms in patients with high inhibitor titers after elective orthopedic surgery (EOS) has, so far, only been addressed in a few publications $[4,11,14,15]$. This case therefore highlights the necessity of including this kind of alternative therapy in the subsequent management of spontaneous hemophilic bleeding complications with regard to ineffective hemostatic cover and to cost reduction.

\section{Case Report}

A 40-year old male patient with severe hemophilia A (factor VIII:C < $1 \%$ ) and high responding inhibitors and with a familial history of hemophilia presented with extensive knee pain, marked fixed flexion deformity of approximately $20^{\circ}$, and a progressive inability to walk. The genetic mutation for hemophilia A could not be identified. An intron 22 inversion, which is commonly seen with severe hemophilia A with high responding inhibitors, was, however, excluded. Since the late 1970s, the patient has been on home treatment and was seen regularly in the Hemophilia Center of our hospital. His clotting factor therapy consisted of factor VIII inhibiting bypassing activity (FEIBA; Baxter AG, Volketswil, Switzerland), porcine factor VIII (Hyate C; Opopharma AG, Glattbrugg, Switzerland), and, for the last 2 years, recombinant activated factor VIIa (rFVIIa; NovoSeven; Novo Nordisk, Bagsvaerd, Denmark). The patient is human immunodeficiency virus (HIV) negative and has an associated transfusion hepatitis B and chronic hepatitis C without any features of liver cirrhosis.

Recurrent joint hemorrhage led to a severe hemophilic arthropathy involving the knees, elbows, and ankle joints. Persistent hemorrhage and associated secondary degenerative disease led to a severe bilateral knee fixed flexion deformity associated with severe pain and progressive difficulty in walking. The patient was hardly able to rise from a seated position. Due to bilateral severe knee disease and hemophilia A with high responding 
inhibitors and in order to restrain costs, bilateral knee replacement surgery at one setting was recommended.

At hospitalization, 4 days preoperatively, the inhibitor level was low with 2 Bethesda units (BU). After a dose of rFVIIa (120 $\mu \mathrm{g} / \mathrm{kg}$ body weight), a central venous catheter was inserted for the delivery of extracorporeal Protein-A immunoadsorption (PAIA) treatment. PAIA was administered on day 3 and day 1 before surgery and three times postoperatively. Unfortunately, there was extensive complicating soft tissue hemorrhage around the catheter site and 10 additional doses of rFVIIa were necessary.

Prior to surgery, the inhibitor titer was not detectable. Factor VIII [a total dose of 10,000 IU (i.e. $100 \mathrm{IU} / \mathrm{kg}$ body weight) Haemate HS; ZLB Behring AG, Berne, Switzerland) was administered immediately before and during surgery. The factor VIII:C level was brought to $124 \%$ and kept above $100 \%$ during surgery and the first 3 postoperative days. TKAs were performed bilaterally under tourniquet control to minimize bleeding at the bone-cement interface. The operation was performed by an anteromedial approach without osteotomy of the tuberosities. In order to mobilize the knees, an extensive adhesiolysis of the dorsal capsule and the collateral ligaments had to be performed. Both components (femoral and tibial) were cemented with an ultracongruent tibial polyethylene plateau (Innex ${ }^{\mathrm{TM}}$; Sulzer AG, Baar, Switzerland), sacrifying the posterior cruciate ligament.

At the end of surgery, the right knee joint had a flexion to $80^{\circ}$ and an extension deficit of $5^{\circ}$ and the left knee had a flexion to $80^{\circ}$ with full extension. Operative time was $1.5 \mathrm{~h}$ per knee joint and there were no complications reported during surgery.

Postoperatively, thrombosis prophylaxis with subcutaneous LMW Heparin was administered for the period of time of factor VIII replacement. On day 4 postoperatively, the inhibitor titer increased and the response to substitution with factor VIII decreased. On the fifth, seventh, and eighth postoperative day, PAIA was administered again in an attempt to reduce the rising inhibitor titer, but without success. The treatment was changed to rFVIIa $(120 \mu \mathrm{g} / \mathrm{kg}$ every $3 \mathrm{~h})$. Despite this aggressive regimen, bleeding into the right knee joint was observed, and on the sixth postoperative day, hemorrhage of the left knee joint was additionally diagnosed. After increasing the therapy with rFVIIa, the hemorrhages stopped. On the 11th postoperative day, the patient complained of new and severe pain within the right knee. Color duplex flow ultrasound (US) suggested the diagnosis of a pseudoaneurysm.

With the patient under local anesthesia, digital subtraction angiography (DSA) via a femoral artery approach was performed using a $5 \mathrm{~F}$ vascular sheath (Terumo, Tokyo, Japan). A 5F pigtail catheter (Cook, Bloomington, IN, USA) was used to obtain a nonselective diagnostic arteriogram to identify the source of the bleeding. Selective angiography was performed with a cobra-shaped catheter (5F, C-2; Cook, Bloomington, IN, USA) in order to localize the feeding artery. Diagnostic catheter maneuvers were usually done using a steerable 0.035-in. guidewire (Radifocus; Terumo, Tokyo, Japan). DSA confirmed the suspected diagnosis of a pseudoaneurysm arising from the inferior lateral geniculate artery (Fig. 1). TAE was then performed using a coaxially placed microcatheter (Progreat; Terumo, Tokyo, Japan) in proximity to the bleeding branch. For TAE, five fibered 0.018-in. platinum microcoils (VortX; Boston Scientific, Natick, MA, USA) with a nominal configured diameter of $2 \times 3 \mathrm{~mm}$ to $2 \times 4 \mathrm{~mm}$ and a stretched length of 22-30 mm were used. Coil detachment was performed by saline injection. Using TAE, bleeding was effectively stopped within 15 min. At the end of the procedure, a closure device (AngioSeal; St. Jude Medical Devices, Minneapolis, MN, USA) was used to allow safe closure of the puncture site.

As a result of TAE, rapid mobilization of the patient with crutches was possible and he was discharged on day 26 . Three months postoperatively at clinical follow-up there was bilateral flexion to $90^{\circ}$ passively and to $80^{\circ}$ actively. There was a $5^{\circ}$ extension deficit bilaterally. Rebleeding did not occur. The patient is now able to walk continuously and unassisted for up to $3 \mathrm{~h}$; in addition, there is less pressure and pain associated with his ankle joints bilaterally. All in all, factor replacement therapy was performed by supplementation of 59,000 IU of factor VIII and $1464 \mathrm{mg}$ of rFVIIa, with carrying costs of approximately $750000 €$.

\section{Discussion}

Bleeding into a "target" joint like the knee is most commonly observed in patients with hemophilia A and is usually due to synovialitis, erosion, joint destruction, debilitating pain, and secondary osteoarthritis, in addition to the primary disorder $[4,11$, 15]. Rarely have pseudoaneurysms of the arteries supplying the knee joint been described in association with end-stage hemophilic arthropathy as the cause of hemarthrosis [4, 15]. With the availability of adequate clotting factor concentrates, elective TKA has developed to a well-accepted and widespread approach in hemophiliacs, even in those with inhibitors [5-8, 11, 16, 17]. TKA in hemophilia A has been shown to reduce pain and to be associated with a general decreased tendency for hemarthrosis and an undisputed improvement in the quality of life with better mobility $[6,11,17]$; however, perioperatively and postoperatively, some evident complications might occur. The most commonly reported complication of total knee replacement in the setting of hemophilic arthropathy is that of early and late infection and late loosening of the prosthesis, the latter two of which have been described as occurring at a higher rate in patients who are seropositive for HIV $[17,18]$. Legroux-Gerot et al. [18] described recurrent hemarthrosis after TKA in 6 of 12 patients with 17 total knee prostheses, with some requiring synovectomy for recurrent hemorrhage. Thomason et al. [5] reported early perioperative knee joint hemorrhage in 3 of 23 TKAs; however, the cause and treatment was not stated.

Arterial injury in terms of exravasations, pseudoaneurysms, and arteriovenous fistulas during or after TKA might account for a minority of limb- or life-threatening complications, with a latency usually ranging from hours to years [2,3]. Etiologically, there seems to be an intrinsic risk of developing these vascular complications due to the close proximity of significant vascular structures and the area of operative manipulation. In this context, vascular laceration or avulsion by inappropriately placed retractors, overextension, or direct penetration from gouging are, by far, the most common mechanisms of arterial damage [1,3]. Aside from hemophilia, other extrinsic risk factors appear to also play an important role in the development of serious vascular injury after TKA [1, 2, 11]. Many authors stressed the fact that peripheral vascular disease (PVD) is a predominant factor for such a complication [2]. Other investigators reported that foreign body infection, prosthetic loosening, intrapelvic migration, the intense heat of polymerization from methyl methacrylate, or repeated trauma by osteophytes, and screw as well as cement erosion were further extrinsic reasons for the occurrence of serious arterial injury [1]. In our patient, it remains speculative if the pseudoaneurysm of the right inferior lateral geniculate artery already existed prior to TKA, because angiographic diagnosis had only been made postoperatively. However, regarding the postoperative follow-up, it is most likely that the pseudoaneurysm of the right inferior lateral geniculate artery had been caused iatrogenically.

Generally, pseudoaneurysm formation complicating TKA in hemophilic arthropathy is rare, with approximately a handful of cases being reported in the world literature [4, 11, 15]. Associated features include a cavity filled with hematoma and surrounded by adventitia, perivascular tissue, and fibrotic tissue deriving from the hematoma $[9,14,15]$.

The definitive strategy for bleeding episodes complicating TKA in hemophilic arthropathy is replacement therapy with clotting factors $[10,11]$. However, bleeding complications in severely 

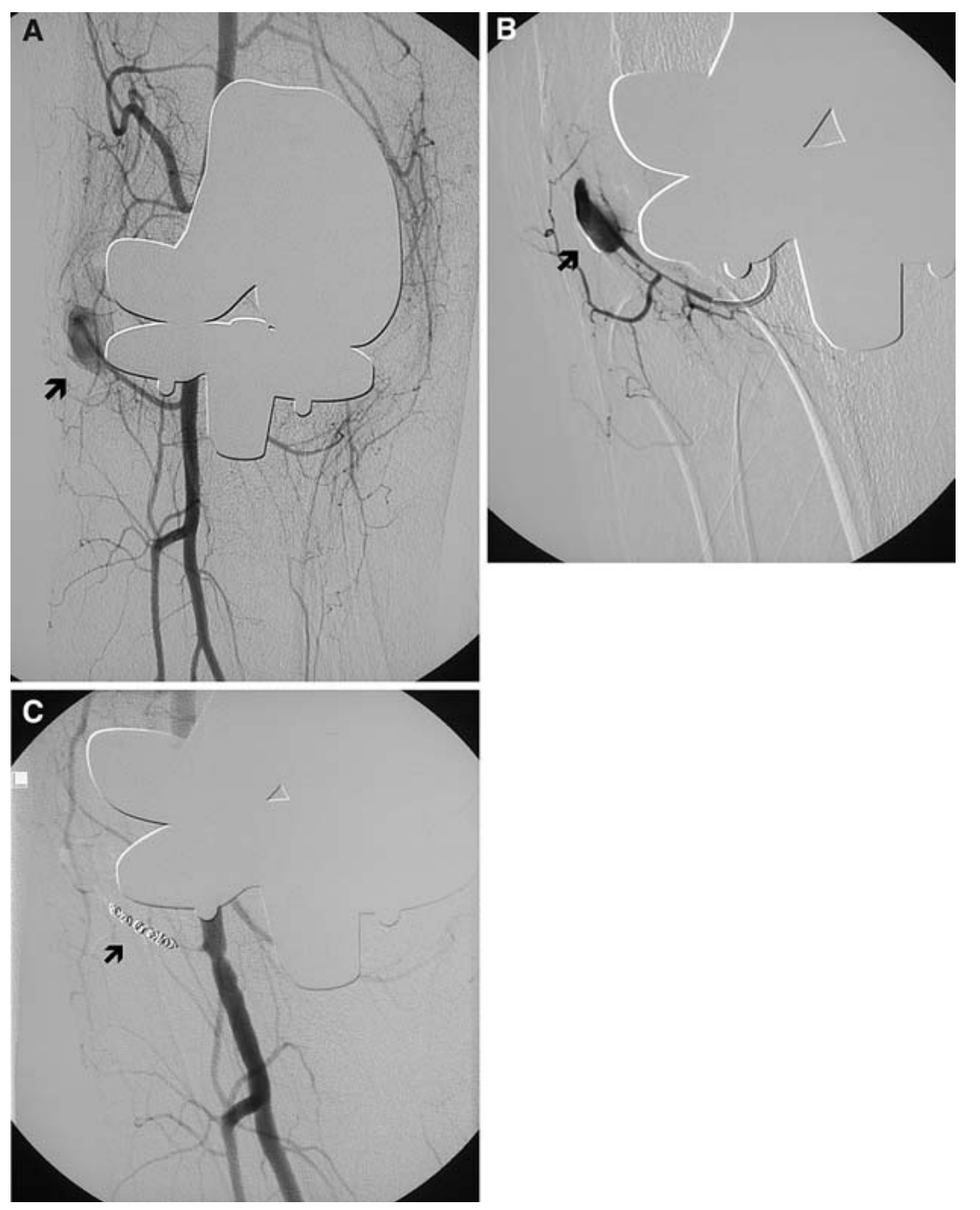

Fig. 1. (A) Selective angiogram obtained in a left oblique projection demonstrates a large pseudoaneurysm of the right inferior lateral geniculate artery (arrow). (B) Superselective angiogram obtained in a left oblique projection by using a $3 \mathrm{~F}$ microcatheter confirms diagnosis of a solitary pseudoaneurysm of the left inferior lateral geniculate artery (arrow). (C) Selective angiogram obtained in a left oblique projection after the successful embolization with microcoils (arrow).

damaged joints or after elective orthopedic surgery are sometimes unresponsive to this kind of treatment, particularly in hemophilia patients with inhibitors [12]. In such situations, an alternative option, especially for the therapy of pseudoaneurysms, is a surgical approach [2, 7, 8]. Surgical treatment usually includes ligation, simple resection of the pseudoaneurysm, closing of the defect with a patch, bypass grafting, end-to-end anastomoses, endaneurysmorrhaphy, and open synovectomy [1, 2, 7]. However, it is important to realize that an open surgical procedure might fail, as the source of bleeding is not always apparent $[1,2]$. Another potential disadvantage of open surgery is that dissection is required in an inflamed area [2]. In addition, surgical interventions might be complicated by deep infection or anatomic distortion from the pseudoaneurysm, or patients might have an increased risk of regional or general anesthetic episodes [13].

With the advent of newer coaxial microcatheter systems and the improvement of embolization techniques, there is an increasing interest in catheter-based treatment as an alternative therapeutic strategy for pseudoaneurysms after TKA, also in hemophiliacs [4, $11,15,19,20]$. It is important to note that with respect to coaxial embolization, acute curving of the microcatheter does not present an obstacle, even for the delivery of microcoils into second- or third-order vessel locations $[1,11,15]$. Some investigators pointed out that percutaneous TAE, carried out as selectively as possible, might improve the chances of success and reduce the risk of complications such as ischemia, as it seems to be less invasive in comparison with a surgical approach $[2,11,19,20]$. Another advantage of a coaxial technique with microcatheter and microcoil systems is that it allows precise localization and catheterization of the bleeding arterial branches [11]. Moreover, this case and the small series from Saris et al. [4] suggest that interventional angiography might have a role when there is clinical concern for a pseudoaneurysm, allowing for contemporaneous diagnosis and therapy. Nevertheless, this procedure at least requires clotting factor replacement therapy for the groin puncture.

Arthroplasty in hemophilic arthropathy is known to be costly, with a mean of $105885 €$ and a range from $56311 €$ to $174907 €$ $[18,21]$; the mean estimated costs are $7045 €$ for surgery, $2345 €$ for the prosthesis, $93392 €$ for clotting factors, and $3174 €$ for rehabilitation. In general, clotting factor replacement might contribute to $88 \%$ of the total cost [18]. However, as seen in our case, the costs for clotting factors might dramatically increase if hemarthrosis occurs postoperatively or during rehabilitation, especially in patients with inhibitors.

In conclusion, because of a complicating pseudoaneurysm of the inferior lateral geniculate artery with hemarthrosis after TKA, 
our patient had to be treated for a longer period of time and at a more intensive level, which proved to be extremely costly. He suffered an extended period of pain and his rehabilitation was markedly prolonged from a usual 2-month period to 3 months, with nearly 4 weeks of hospitalization. Initially, hemarthrosis after TKA was thought to be caused by the patient's primary disorder and was, therefore, treated by clotting factors. After clotting factor replacement therapy had failed, an alternative diagnosis was thought to be causative for excessive bleeding and severe pain. Angiography with embolization represented a minimally invasive interventional technique to efficiently diagnose and treat the pseudoaneurysm formation. Radiologists as well as clinicians should be aware of this cost-effective modality that requires close therapeutic alliance in order to advance the standard of care for hemophiliacs. In this context, they should also keep in mind that pseudoaneurysms are extremely infrequent conditions that might not only occur after TKA but also without previous surgery in hemophilia patients; for that reason, preoperative angiography is recommended in these special patients. On the basis of these facts, this case illustrates that a timely intradisciplinary approach to diagnosis and treatment is of utmost importance and that there is a need for regular conjoint conferences and meetings.

\section{References}

1. Barriga A, Valenti Nin JR, Delgado C, et al. (2001) Therapeutic embolisation for postoperative hemorrhage after total arthroplasty of the hip and knee. J Bone Joint Surg Br 83:90-92

2. O`Connor JV, Stocks G, Crabtree JD Jr, et al. (1998) Popliteal pseudoaneurysm following total knee arthroplasty. J Arthroplasty 13:830832

3. Ibrahim M, Booth RE Jr, Clark TWI (2004) Embolization of traumatic pseudoaneurysmy after total knee arthroplasty. J Arthroplasty19:123128

4. Saris DBF, van Rinsum AC, Dhert WJA, et al. (1997) Periarticular aneurysm formation in haemophilia. Lancet 349:766-768

5. Thomason HC 3rd, Wilson FC, Lachiewicz PF, et al. (1999) Knee arthroplasty in hemophiliac arthroplasty. Clin Orthop 360:169-175
6. Tagariello G, Bisson R, Radossi P, et al. (2003) Concurrent total hip and knee replacements in a patient with haemophilia with inhibitors using recombinant factor VIIa by continuous infusion. Haemophilia 9:738-740

7. Rodriguez-Merchan EC, Wiedel JD, Wallny T, et al. (2004) Elective orthopedic surgery for hemophilia patients with inhibitors: New opportunities. Semin Hematol 41(Suppl 1):109-116

8. Hvid I, Rodriguez-Merchan EC (2002) Orthopaedic surgery in haemophilic patients with inhibitors: An overview. Haemophilia 8:288-291

9. Scoles PV, King D (1980) Traumatic aneurysm of the descending geniculate artery: A complication of suction drainage in synovectomy for hemophilic arthropathy. Clin Orthop 149:153-159

10. Young G, McDaniel M, Nugent DJ (2005) Prophylactic recombinant factor VIIa in haemophilia patients with inhibitors. Haemophilia 11:203-207

11. Mauser-Bunschoten E, Zijl JAC, Mali W, et al. (2005) Succesful treatment of severe bleeding in hemophilic target joints by selective angiographic embolization. Blood 105:2654-2657

12. Scharrer I (1999) Recombinant factor VIIa for patients with inhibitors to factor VIII or IX or factor VII deficiency. Haemophilia 5:253-259

13. Thomas CD, Asumu T, Chalmers N, et al. (2000) Therapeutic embolization for sustained hemorrhage following total hip replacement in a patient with hemophilia A. Orthopedics 23:849-850

14. Keller A, Terrier F, Schneider P-A, et al. (2002) Pelvic haemophilic pseudotumour: Management of a patient with high level of inhibitors. Skeletal Radiol 31:550-553

15. Mann HA, Goddard NJ, Lee CA, et al. (2003) Periarticular aneurysm following total knee replacement in hemophilic arthropathy. J Bone Joint Surg Am 85:2437-2440

16. Rodriguez-Merchan EC (1998) Management of orthopaedic complications of hemophilia. J Bone Joint Surg Br 80B:191-196

17. Cohen I, Heim M, Martinowitz U, et al. (2000) Orthopaedic outcome of total knee replacement in haemophilia A. Haemophilia 6:104-109

18. Legroux-Gerot I, Strouk G, Parquet A, et al. (2003) Total knee arthroplasty in hemophilic arthropathy. Joint Bone Spine 70:22-32

19. Pisco J, Garcia V, Martins M, et al. (1990) Haemophilic pseudotumor treated with transcatheter arterial embolization: Case report. Angiology 41:1070-1074

20. Sevilla J, Alvarez M, Hernandez D, et al. (1999) Therapeutic embolization and surgical excision of haemophilic pseudotumor. Haemophilia 5:360-363

21. Beeton K (2002) Evaluation of outcome of care in patients with haemophilia. Haemophilia 8:428-434 